Basic Health Sciences

Poster

Abstract ID: 62

\title{
Anatomical variations of the anterior communicating artery complex: a multidetector CT angiographic study
}

Soe Ei Phyu ${ }^{\mathrm{a}}$ | Zunariah Buyong ${ }^{\mathrm{a}}$ | Radhiana Hassan ${ }^{\mathrm{b}}$ | Jamalludin A. Rahman ${ }^{\mathrm{c}}$ | Siti Kamariah Che Mohamed $^{\mathrm{b}}$

${ }^{a}$ Department of Basic Medical Sciences, Kulliyyah of Medicine, International Islamic University Malaysia

${ }^{b}$ Department of Radiology, Kulliyyah of Medicine, International Islamic University Malaysia,

'Department of Community Medicine, Kulliyyah of Medicine, International Islamic University Malaysia

Introduction: The anterior communicating artery (ACOA) complex of the cerebral circulation, an area with great anatomical diversity, forms part of the communicating arterial supply to the brain. As brain tissues are susceptible to ischemic death, knowledge of this variability is important in diagnosis and management of diseases affecting brain circulation. The aim of the study is to measure the prevalence and to describe these variations. Methods: All patients who underwent CT angiography (CTA) scanning in HTAA from January 2009 to August 2015 were selected. A cross-sectional study was done to study these variations in 81 reconstructed CTA images. Results: Eleven types of variations were described (typical pattern; hypoplasia, aplasia, and duplication of ACoA; hypoplasia, and aplasia of $A 1$ segment of the anterior cerebral artery (ACA); hypoplasia, and aplasia of $A 2$ segment of ACA; $A 2$ segments of ACA arising from a common trunk; the third $A 2$ segment; bihemispheric ACA). A1 segment is the part of ACA from the internal carotid artery to ACoA, and $\mathrm{A} 2$ segment is the part of ACA from ACoA to the junction between the rostrum and genu of the corpus callosum. The typical pattern was seen in $35.8 \%$, and the cases with other variation types constituted $64.2 \%$, which is higher compared to previous studies. The variations in $\mathrm{ACoA}$ alone were $43.2 \%$, and the most common variation was the ACoA aplasia, accounting for $28.4 \%$. Conclusions: This study shows the high prevalence of anatomical variations in the ACoA complex, and the probable difference of this figure in different populations.

KEYWORDS: Cerebral supply, anterior communicating artery complex, Circle of Willis 